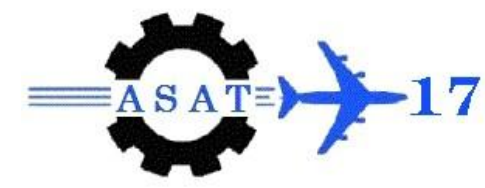

\title{
A Spatiotemporally Variable Model for Nowcasting Storm Motion Vectors using Remotely Sensed Raster Data
}

\author{
Shaimaa El Sharkawy ${ }^{*}$, Mona Safar ${ }^{\dagger}$, Mohamed A. Gad ${ }^{\ddagger}$
}

\begin{abstract}
This paper presents a tracking and forecasting model that performs both pattern tracking and forecasting of the motion fields of the rainfall patterns that are detected using raster-based remote sensors (weather satellites and radars). The technique uses a distributed version of the cross correlation idea on subsets of the subsequent images to determine the velocity field at any time step. The velocity field obtained from the subsets is spatially interpolated to the pixel level to determine a high resolution version of the velocity field. The forecasting part implements a novel idea of using an exponential filter with parameter updating to adaptively fit the temporal evolution of the velocity vectors at every pixel. The effectiveness of the model is illustrated using Meteosat Images. We were able to effectively track and forecast the velocity vectors of the cloud patterns at every pixel of the Meteosat extent. Our initial experience indicates that the developed model shall benefit many application domains.
\end{abstract}

Keywords: Rainfall; Weather; Clouds; Forecasting; Nowcasting; Satellite; Radar; Vector.

\section{Introduction}

Rainfall fields contain a complicated mixture of cloud structures which are moving in different directions, developing and dissipating, come close to each other or go apart and, doing so, move across the catchment or a region. Rainfall patterns occur on different spatial and temporal scales. These scales are usually classified into four main categories: the microscale, mesoscale, synoptic scale, and the climatological scale. The classification is based on the spatiotemporal resolution (i.e., the size of the calculation grid cell) and the spatiotemporal scale (the extent of the calculation domain). Each category is also subdivided into subcategories [1]. Clouds and precipitation meso scale systems represent some of the most important and scientifically exciting weather system in, these systems produce heavy rains, severe winds including downbursts and tornadoes, hail thunder and lightning and major snow storms. Forecasting such storms is a major challenge since they are too small to be adequately resolved by conventional observing networks and numerical prediction models. The whole forecasting process involves the determination of the current state of the process (tracking) as well as what shall happen in the future (forecasting). This study is concerned with precipitation estimation and forecasting on the meso- $\gamma$ scale (4 Km spatial, and 30 minutes' temporal resolution).

Graduate Student, Civil Eng. Dept., Ain Shams Univ., Egypt; shaimaa.elsharkawy@gmail.com

Assistant Professor, Computer and Systems Engineering Dept., Ain Shams Univ., Egypt;

mona.safar@eng.asu.edu.eg

* Associate Professor, Civil Eng. Dept., Ain Shams Univ., Egypt, hydroshams@yahoo.ca 
Precipitation measuring is a complicated meteorological observation because precipitation occurs intermittently and with high spatial and temporal variability. Remote sensed data offers a continuous description of atmospheric parameters from the synoptic to regional scales. The main sources of remote sensing data for rainfall and clouds are weather radars and weather satellite. The products of the two sensors are always in grid formats (either image or binary grids). Radar data is a ground-based measurement since the radar is located on the ground. Meteorological radar has the advantage of measuring the rainfall rate. Satellite data, though cannot measure rainfall intensity directly, it can provide a bigger picture of what is happening in the atmosphere in terms of the existence of cloud systems. Weather satellite data is space measurements since the sensor is located in space. There are two types of weather satellite: the polar orbiting satellites and the geostationary satellites. This study focuses on data from the geostationary weather satellites due to its higher temporal resolution and the ability to generate and animate a sequence of images. The Geostationary satellites (an active sensor) is located at a height of approximately $36000 \mathrm{~km}$ above the equator. The satellite rotates in an orbit parallel to the equator with a certain speed so that the satellite is always fixed (i.e., stationary) with respect to a point on the surface of the earth. This enables the continuous monitoring of the same face of the earth. This continuous monitoring of the same part produces a high temporal resolution (commonly 15-30 min between acquisitions). The product is in image formats. The images include three main channels: (1) visible, (2) (IR) infrared image, and (3) water vapor. The visible image is the equivalent of taking a black and white photo of the earth from space. The bright areas show where the sun is being reflected back into space as a result of clouds or snow cover. Clouds and snow show up white. The thicker the cloud, the brighter the white color. Land surfaces show up as gray and ocean surfaces nearly black. The major limitation to visible imagery is that it is only valid during daylight. The (IR) infrared image shows heat based radiation from the infrared spectrum. In other words, the warmer the surface, the more infrared radiation it emits. In a satellite IR image, cooler surfaces are bright and warmer surfaces are dark. Since the atmosphere cools as altitude increases, clouds would show up as bright areas and land surfaces as dark areas. In addition, low clouds will be grayer and higher clouds will show up more white. Tall thunderstorm clouds will show up as bright white. A large advantage of IR is that it is available twenty-four hours a day. Satellite data is a valuable source of information for operational forecasters. It is being used as an analysis tool, direct aid to short period forecasts (6-12 hours ahead of cloud, rainfall, floods etc., and is also used as input to NWP (numerical weather prediction models) for defining initial condition.

Continuous and reliable global precipitation information is crucial for countless applications ranging from weather and climate to people daily life. Severe weather and heavy rainfall events exact a heavy toll in both lives and property damage. Hydro-meteorologists are trying very hard each day to provide accurate weather information. There is an actual attention in the forecasting of rainfall at very short lead-times (Nowcasting). Nowcasting is very vital in many fields such as severe storms warning systems and flash flood forecasting, aviation safety, realtime operation of flood control structures (e.g., dams), real-time management of urban drainage systems and producing CMV (cloud motion vectors) required to update the NWP models (Numerical Weather Prediction Models). Nowcasting is defined as short-term weather forecasting with forecast lead times up to six hours with high accuracy. Nowcasting is not a new concept, it was devised in the mid-1970s [2], a detailed book on the subject was published a few years later [3]. Nowcasting techniques are usually based on methods of weather extrapolation capable of producing 6-hrs (or less) forecasts that are higher in accuracy than NWPMs (Numerical Weather Prediction Models). NWPMs are typically run over large spatial scales and at low spatial and temporal resolutions as compared to nowcasting techniques [4]. NWPM resolutions do not meet the user requirements for the realtime flood prediction applications for several reasons. The low spatial resolution $(30-100 \mathrm{~km})$ 
of a NWPM output is often so large that it misses sub-grid scale processes such as small fronts or convective activity. The relatively infrequent temporal spacing of forecasts (6-24 hours) of NWPMs can only provide general guidance about the large-scale state of the weather at the forecasted time. Also the complexity of the algorithms is computationally very demanding and creates another drawback, as the spin-up and adjustment times for these models are often on the scale of 6 hours [4 -7]. Hence, NWPMs are usually being operated using large supercomputers at the governmental level. Figure (1) shows a comparison of the accuracy of the forecasts for the different models scales. Note that nowcasting models produce superior forecasts up to lead times 3 hours and the NWP models are better for 6-36 hours lead times. Hence, the most accurate forecasts for short lead-times (zero to six hours) are produced by extrapolation methods [26]. It should also be noted that nowcasting techniques are actually used to develop the cloud motion fields required to calibrate and update the state of the NWPMs.

The research on automatic storm identification, tracking and forecasting on the nowcasting level has been in progress for half a century using remote sensing data. Since 1960s, the availability of real time radar and satellite observations provided the basis for very short range weather forecasting by extrapolation algorithms (i.e., nowcasting). Several algorithms have been adopted. These algorithms are usually based on either the feature tracking concept to individually detect and track storms [13 -17 and others] or cross correlation to define the storms motions for a portion or a whole image [4, 8, 12, 18, 29 and others]. The feature tracking concept depends first on isolating patterns then tracking the pattern features (e.g., centroid, vertices, or intensity contours). The feature tracking concept can suffer mismatching problems in case of interfering patterns in which storms cannot be isolated separately (i.e., which feature belongs to which storm in subsequent images). The cross correlation technique, our proposed technique, can overcome these mismatching problems, since it depends on correlating the whole or portions of the satellite/radar domain with subsequent scans. This correlation procedure can take into account the detailed shape of the pattern being tracked, and decreases the chances of mismatching patterns. Hilst and Russo [19] were the earliest attempt in tracking and forecasting of radar rainfall patterns via correlation analysis. Also, Noel and Fleischer [20] are among the earliest radar meteorologists to examine the predictability of precipitation echoes using correlation analysis. More remarkable papers are those published by Russo and Bowne [21] and Kessler and Russo [22]. Kessler [23] and Wilson [8] explored the use of cross correlation methodology to determine a best estimate of echo pattern average movement. The 1970s saw more development on cross correlation analysis for nowcasting algorithms. Leese used consecutive satellite images to produce a "distributed" vector field based on the correlation of portioned cloud images, this was one of the first attempts to determine a spatially variable velocity field [24]. Zawadzki [9] developed an optical device to measure the space-time statistical properties of radar inferred precipitation fields. Following these trials, Austin and Bellon [10] employed the correlation methodology for single radar forecasting, they developed an automated pattern matching program correlating whole radar images for nowcasting precipitation up to 3 hours ahead. A landmark development has been made by Rinehart and Garvey [25], they used the tracking of TREC (Radar Echoes with Correlations) algorithm to estimate a full grid of spatially varying velocities based on correlating small sub-divisions of radar reflectivity images. The 1990s saw the first attempts to run convection resolving NWP model forecasts assimilating radar data [27]. Also, the use of successive GOES satellites images combining with correlation analysis to estimate cloud motion vectors provided tangible development in quality control and interpolation. Hamill and Nehrkorn [5] developed a basic cloud motion forecasting model using lag cross correlations, the model suffers of inability to deal with any significant changes in the velocity vectors inside the same rainfall patterns (i.e., differential motion). Gad [28] 
developed a new radar-based model for quantitative short-term forecasting of rainfall fields. The model employs also the cross correlation tracking idea and an optimization strategy for performing the cross-correlation analysis to reduce the run time significantly and make the technique attractive for real-time applications. In recent years, specifically at (2012), a new nowcasting algorithm is introduced for short-term high-resolution precipitation forecasting [30]. In 2016, a new 3D of a COTREC (continuity of TREC)-based algorithm of precipitation tracking system was developed using a new-generation weather radar [31]. However, the new model is still based on the same theory of the TREC old model.

In summary, and to the best of our knowledge, the available literature for nowcasting techniques indicates that most techniques suffer from two main problems: (1) the inability to deal with differential motion (i.e., develop a separate velocity vector for each high resolution satellite pixel, and (2) the forecasts are made using advection which can produce significant errors since the motion is actually very nonlinear (usually spiral across the pressure contours of the low pressure systems). Our paper here tries to overcome the two problems in order to fill this gap.

\section{Problem Formulation}

It can be concluded that the problem of tracking/forecasting cloud patterns (i.e., nowcasting applications) is performed on two sequential stages: the tracking stage and the forecasting stage. The major problems that generally face nowcasting techniques are the spatiotemporally variability of the rainfall patterns being tracked/forecasted which can lead to mismatching in tracking and errors in forecasting, in addition to the computational demands and run-time requirements. Accordingly, the problems facing this research can be summarized in the following three challenges:

- How to deal with differential motion (the clouds can have different motion directions inside the same pattern). In other words, how to develop a separate velocity vector for each high resolution satellite pixel.

- Finding a more stable forecasting (projection) approach that is less sensitive to the variable nature of the clouds patterns.

- How to deal with the non-linear motion (usually spiral).

This paper presents a tracking and forecasting model that overcomes these problems (i.e., challenges) in order to perform both pattern tracking and forecasting of the CMV (cloud motion vectors). The tracking part of the developed model is based on the cross correlation concept on portions of the satellite domain while a novel idea is presented in eth following sections for the forecasting part.

\section{Methodology}

The model developed in this paper is called CMDF (Cloud Motion Detector and Forecaster). CMDF consists of two main components: (1) CMD (Cloud Motion Detector) and (2) CMF (Cloud Motion Forecaster). Description of the two components follows: 


\subsection{CMD (Cloud motion vectors detector)}

\subsubsection{X-Correlation Detection}

Motion detection is performed by cross correlating every two successive satellite images. Figure (2) depicts the methodology of cross-correlation. The satellite domain is divided into number of square boxes (subsets) as specified by user and the matching is performed in each square box separately. Figure (3-a) presents how the satellite domain is subdivided into subsets (boxes). The CMD module works as follows for each box:

1. Define the extent of a certain box $\mathrm{k}$.

2. Retrieve the array of values from the first image (at time t-1) inside the defined extent (denoted Grid(t-1)).

3. A quality query is made on the box $\mathrm{K}$ of Grid(t-1) to check variability inside (any box that has a coefficient of variation CV (standard deviation / mean) $<0.2$ is excluded from the analysis. This filter is very important to exclude black (no cloud boxes) or very white boxes that has no distinguished patterns to correlate.

4. Shift this extent by any grid length ( $\mathrm{dx}$ and $\mathrm{dy}$ ) and retrieve the array of values from the next image (at time $t$ ) inside the shifted extent (denoted Grid(t)).

5. Calculate the correlation coefficient $(\rho)$ between $\operatorname{Grid}(\mathrm{t}-1)$ and Grid(t) as follows:

$$
\rho_{\mathrm{dx}, \mathrm{dy}}=\frac{\frac{1}{\mathrm{~N}} \sum_{\mathrm{i}=1}^{\mathrm{i}=\mathrm{N}}\left(\mathrm{F}_{\mathrm{i}, \mathrm{t}-1}-\overline{\mathrm{F}}_{\mathrm{i}, \mathrm{t}-1}\right)\left(\mathrm{F}_{\mathrm{i}, \mathrm{t}}-\overline{\mathrm{F}}_{\mathrm{i}, \mathrm{t}}\right)}{\sigma_{\mathrm{t}-1} \sigma_{\mathrm{t}}}
$$

where:

- N: Number of pixels in any of the two consecutive grids (i.e., dimension of the extent).

- $\mathrm{F}_{\mathrm{i}, \mathrm{t}-1}$ :image value at pixel $\mathrm{i}$ from the first image.

- $F_{i, t}$ : image value at pixel $j$ from the second image. Note that $x j=x i+d x$ and $\mathrm{yj}=\mathrm{yi}+\mathrm{dy}$

- $\overline{\mathrm{F}}_{\mathrm{i}, \mathrm{t}-1}$ and $\sigma_{\mathrm{t}-1}$ : the average and standard deviation of the image values inside Grid(t-1).

- $\overline{\mathrm{F}}_{\mathrm{i}, \mathrm{t}}$ and ${ }^{\sigma_{t}}$ : the average and standard deviation of the image value inside $\operatorname{Grid}(\mathrm{t})$.

6. Repeat steps 4 and 5 until maximum correlation $\left(\rho_{\max }\right)$ is obtained. The optimum spatial shift $\left(\mathbf{d x}_{\mathbf{k}, \mathbf{t}}, \mathbf{d y}_{\mathbf{k}, \mathbf{t}}\right)$ and the corresponding velocity vector $\mathbf{V}_{\mathbf{k}, \mathbf{t}}$ (i.e., at box $\mathrm{k}$ and at time t) are those that maximize the correlation coefficient between Grid(t-1) and $\operatorname{Grid}(\mathrm{t})$. Hence, the velocity vector at box $\mathrm{k}$ and at time $\mathrm{t}$ is given by:

$$
\mathrm{V}_{\mathrm{k}, \mathrm{t}}=\left\{\frac{\mathrm{dx} \mathrm{k}, \mathrm{t}}{\Delta \mathrm{t}}, \frac{\mathrm{dy} \mathrm{y}_{\mathrm{t}, \mathrm{t}}}{\Delta \mathrm{t}}\right\}
$$

where $\Delta \mathrm{t}$ is the temporal separation between the two satellite images which is usually 30 minutes. Note that if the first grid $(\operatorname{Grid}(\mathrm{t}-1))$ is shifted by the obtained velocity vector, the two grids (Grid(t-1) and Grid(t)) will have best match. Figure (3-b) presents how to the CMD module works on every two successive satellite images.

\subsubsection{Velocity Vectors Interpolation}

Once the velocity vectors are determined at the center of each subset (box), spatial interpolation using the IDW (inverse distance weighted) technique is implemented to interpolate the calculated vectors to each pixel of the satellite domain. Let $\mathrm{V}_{\mathrm{k}, \mathrm{t}}$ denotes the 
velocity vector at the center of box $\mathrm{k}$, the velocity vector at any pixel $\mathrm{i}$ of the satellite domain $\mathrm{V}_{\mathrm{i}, \mathrm{t}}$ can be obtained from:

$$
\mathrm{V}_{\mathrm{i}, \mathrm{t}}=\frac{\sum_{\mathrm{k}=1}^{\mathrm{k}=\mathrm{nbox}} \frac{\mathrm{V}_{\mathrm{k}, \mathrm{t}}}{\mathrm{L}^{2} \mathrm{ik}}}{\sum_{\mathrm{k}=1}^{\mathrm{k}=\mathrm{nbox}} \frac{1}{\mathrm{~L}^{2} \mathrm{ik}}}
$$

where:

- $\mathrm{L}_{\mathrm{ik}}=$ Distance between pixel $\mathrm{i}$ and the center of box number $\mathrm{k}$

- $n$ box $=$ Number of nearest boxes used for the interpolation (taken $=4$ for intermediate pixels to ensure a box at each side and 3 at the border pixels to avoid extrapolation effects).

It should be noted that the IDW interpolation is chosen for this task since the interpolated value is actually a weighted (distance weighted) version of the surrounding vectors which conforms well to the nature of atmospheric circulations which is usually cyclonic. This way, the interpolated velocity field will capture the changes in the intermediate vectors (i.e., differential motions). Accordingly, a high resolution version of the velocity field capturing differential movements is obtained. Figure (4) explains the velocity vectors interpolation process. Figure (5) presents a flow chart describing the details of the CMD module.

\subsection{CMF (Cloud Motion Vectors Forecaster)}

This research presents a novel idea to perform the forecasting part. The first step in developing a forecasting algorithm is to select a model that can best describe the evolution of the process. The process being modeled here is a continuous process that simulates the velocity field of the cloud patterns. The changes in the velocity field at any stationary point in the atmosphere always follow smooth transitions (i.e., changes) and never experience sharp or spiky changes. For example, upper air wind at a fixed location will not change from east direction to the north direction without passing through the north-east direction. Accordingly, the chosen model is a deterministic stationary polynomial function in the time $t$. It should be noted also that this polynomial has been observed in the presence of additive random errors [32] which makes the model less sensitive to any data acquisition errors. In other words, the forecasting algorithm assumes that the velocity vector at a certain stationary point in the atmosphere will change (at the same point) following a non-linear behavior that can be simulated using a polynomial over time. The detailed description of the forecasting algorithm follows.

\subsubsection{Forecasting Methodology}

Let $\mathrm{V}_{(\mathrm{t})}$ denote velocity vector at a certain grid cell at time $\mathrm{t}$. The chosen model can be formulated in a differential form as:

$$
\frac{\mathrm{d}^{3}}{\mathrm{dt}^{3}} \mathrm{~V}_{(\mathrm{t})}=0
$$

Let $\mathrm{A}_{(\mathrm{t})}$ denotes the state of the velocity vector at the grid cell at a certain time $\mathrm{t}$. The state at time $t$ at this grid cell is:

$$
A_{(t)}=\left[\begin{array}{l}
A o \\
A 1 \\
A 2
\end{array}\right]=\left[\begin{array}{cc}
V_{(t)} \\
\frac{d}{d t} & V_{(t)} \\
\frac{d^{2}}{d^{2}} & V_{(t)}
\end{array}\right]
$$


The state $\mathrm{A}_{(\mathrm{t})}$ can be seen as the Taylor series expansion coefficient vector of the polynomial. Hence, the forecasting solution can be written in the vector form as following:

$$
\mathrm{A}_{(\mathrm{t}+\Delta \mathrm{t})}=\mathrm{M}_{\Delta \mathrm{t}} \times \mathrm{A}_{(\mathrm{t})}
$$

where $A_{(t+\Delta t)}$ is the forecasted state using forecasting lead time $\Delta t$ and $M_{(\Delta t)}$ is called the transition matrix and is defined as:

$$
\mathrm{M}_{\Delta \mathrm{t}}=\left[\begin{array}{ccc}
1 & \Delta \mathrm{t} & \Delta \mathrm{t}^{2} / 2 \\
0 & 1 & \Delta \mathrm{t} \\
0 & 0 & 1
\end{array}\right]
$$

Equation (6) is called the transition equation. Once the estimation of the state $A_{(t)}$ at the current time $t$ is obtained, the forecasted state $\mathrm{A}_{(\mathrm{t}+\Delta t)}$ after lead time $\Delta \mathrm{t}$ can be obtained by the transition equation. The forecasting methodology is applied on a per cell-basis, i.e., the velocity vector $\mathrm{V}_{(\mathrm{t})}$ can be seen as a matrix of nrows $\times$ ncols observations representing the gridded velocity vectors where nrows is the number of rows and ncols is the number of columns of the satellite grid. The state $A_{(t)}$ can be seen also as a 3-elements vector where each element is a matrix of dimensions nrows $\times$ ncols.

\subsubsection{Real-Time Parameter Estimation}

An adaptive exponential smoothing scheme [33] to estimate the model's parameters is employed for parameters estimation. The scheme employs the concept of negative feedback where the new forecast is adjusted for the error committed in the previous forecast while allowing the option for varying the relative weight given to recent versus past observations. The real-time parameter estimation procedure amounts to the estimation of the state, i.e., Taylor series expansion coefficients of the polynomials representing the evolution of the velocity vector at the grid cells (i.e., pixels). The adaptive exponential smoothing scheme puts more weight on the most recent observation so the influence of observation errors on the estimation decreases rapidly with the age of the observation.

To explain this, suppose that the satellite domain consists of nrows $\times$ ncols grid cells, where nrows is the number of rows and ncols is the number of columns of the satellite grid. Accordingly, a number of nrows $\times$ ncols independent filters can be used to estimate the statevariables of the grid cells. These filters follow the same mathematical form but are completely disconnected. Thus, only one filter is described in here. Considering the theory of adaptive exponential smoothing (Brown, 1963), the updated state at the current time $A_{(t+\Delta t)}$ can be estimated from the previous state $\mathrm{A}_{(\mathrm{t})}$ by:

$$
\mathrm{A}_{(\mathrm{t}+\Delta \mathrm{t})}=\mathrm{M}_{(\Delta \mathrm{t})} \mathrm{A}_{(\mathrm{t})}+\mathrm{H}^{\prime} \mathrm{E}_{\mathrm{t}}
$$

where $\mathrm{H}^{\prime}$ is the smoothing vector and $\mathrm{E}_{(\mathrm{t})}$ is the error vector and are given by:

$$
\begin{aligned}
H^{\prime} & =\left[\begin{array}{c}
1-\left(1-\beta^{\prime}\right)^{3} \\
\frac{3}{2} \beta^{\prime 2}\left(2-\beta^{\prime}\right) \\
\beta^{\prime 3}
\end{array}\right] \\
\mathrm{E}_{(\mathrm{t})} & =\mathrm{V}_{(\mathrm{t}) \text { actual }}-\mathrm{V}_{(\mathrm{t}) \text { forecast }}
\end{aligned}
$$


where:

- $A_{(t)}$ : Updated state-vector at the previous time step. This state-variable will be used for forecasting the next forecast (at time $t+\Delta t$ ) using the transition equation.

- $\mathrm{A}_{(\mathrm{t}+\Delta \mathrm{t})}$ : State-variable at time $\mathrm{t}+\Delta \mathrm{t}$.

- $\mathrm{H}^{\prime}$ : Smoothing coefficient vector.

- $E_{(t)}$ : The error between the observed velocity vector at time $t$ (i.e., $\mathrm{V}_{(\mathrm{t})}$ observed) and the t-step ahead forecast of the velocity vector (i.e., $V_{(t)}$ forecast)

- $\quad \beta^{\prime}$ : Exponential smoothing coefficient.

The values of the $M(\Delta t)$ matrix and the $H^{\prime}$ vector are set in the initialization of the model. They are then kept fixed throughout the forecasting operation. The choice of the value of the exponential smoothing coefficient $\beta^{\prime}$ is based on the stability of the rainfall field. If the evolution of the rainfall field being observed on the satellite scans is very unstable, a smaller value of $\beta^{\prime}(0.1-0.3)$ should be chosen so that more weight is put on older observations. Although this makes the algorithm less sensitive to rapid changes, smoother estimates will be obtained in the presence of small scale noise. If the rainfall pattern is stable, a larger $\beta^{\prime}(0.3-$ 0.5 ) can be taken to make the algorithm respond quickly to changes. Also, for long lead time forecasting $\left(60,120\right.$ minutes and above), a smaller value of $\beta^{\prime}(0.05-0.15)$ should be chosen to give more weight on older observations to avoid overestimating or underestimating the velocity vectors due to the effect of quick responses to newer observations on the long term extrapolation.

In the initialization of the model and in the case of newly appearing storm cells or disappearing cells, the initial values of the state-vector are set by neglecting the higher order terms. For example, for the first time step or for the first time a storm appears, $A_{o}$ is set to the observation value $V(1)$ at time 1 , while $A_{1}$ and $A_{2}$ are set to zeros. For the second time step, $A_{o}$ is set to $\mathrm{V}(2), A_{1}$ is set to $(\mathrm{V}(2)-\mathrm{V}(1))$ or simply to zero, and $A_{2}$ is set to zero, and so on. In subsequent time steps, the updated estimates of the state-vector are then obtained directly from equation (8). Figure (6) presents a flow chart describing the details of the CMF module

\section{Case study}

The model is applied here on satellite images acquired by geostationary satellites, the following subsections present the data-preprocessing, input, output, and an assessment of the model performance.

\subsection{Data Pre-processing}

The pre-processing of the data involves two or three steps (depending on how many satellites are involved) that were automated using GIS (Geographic Information System) as follows:

1. The first step is to geo-reference the images into near-vertical satellite perspective projection (i.e., convert the coordinates from image coordinates into real-world coordinates in a satellite near-vertical perspective projection). This is done by selecting fixed four control points om the satellite image with known real-world coordinates then rectify the image accordingly. Figure (7) shows a schematic representation of the satellite near-vertical perspective projection. If more than one satellite is involved, then the images are geo-referenced each in its different corresponding satellite perspective plan of projection. 
2. The second step applies only if more than one satellite is involved. In that case, the geo-referenced images from the different satellites perspective plans are projected into a full-earth common coordinate system. Examples of full-earth common coordinate systems is 1984 (WGS84) Geographic World Geodetic System or Mercator projection. Figure (8-a) shows two sample images from GEOS-12 and MTSAT after geo-referencing them each in its separate satellite near-vertical perspective coordinate system while Figure (8-b) shows the same two images after projecting them into a common WGS84 coordinate system.

3. Finally, the images formats are converted into standard grid formats (ASCII or Binary) to facilitate reading the pixel values into the model C-Arrays.

\subsection{Real-Time Model Application}

The severe storm that occurred on November 2 and 3, 1994 and caused severe floods in Upper Egypt and Sinai Peninsula is selected here to present the functionality of the developed model. Meteosat-9 images were pre-processed as in section 6.1 above. Note that only Meteosat-9 satellite is sufficient since the focus here is Egypt and the surrounding area and no need to involve other satellites. Accordingly, the coordinate system used is Meteosat-9 near-vertical satellite coordinate system. The data set is 96 images covering the two days. Figure (9) presents a sample sequence of pre-processed grids during this storm.

The objective here is to imagine as if the model is being run in real-time when this storm occurred. To achieve so, the tracker is run first "in off-line mode" to determine the cloud motion vector field between every two successive images. The forecasting is then initialized with the first image and imagined to be run in real-time (i.e., the forecaster is updated at every time step with the new cloud motion vector as if it is newly acquired in real-time). In other words, at every time step, the model is updated with each new image and produces the required cloud vector forecasts. Figure (10-a) shows sample two successive images $(a, b)$ and the calculated velocity field (c) resulted from CMD module. Figure (10-b) provides visual comparison between the actual velocity vectors and the forecasted velocity vectors that have been forecasted in the past using 30 and 120 minutes forecasting lead times (i.e., forecasts issued 30 and 120 minutes ago). As shown on the figures, the model forecasts of the velocity vector fields agree well with the actual velocity vector fields (up to 120 minutes lead time) which gives rise to the operational applicability of the model under this forecasting lead times.

\subsection{Performance and Accuracy Assessment}

1. The whole model is written using $\mathrm{C}++$, instead of high level programming packages in order to achieve the best computational performance. The run times is very acceptable (few seconds) for single satellite coverage. It should be noted that Run time varies according to the hardware capabilities upon which it is executed. The code is automated to be implemented to the whole sample of the successive grids.

2. In order to fully evaluate the forecasting accuracy. Ruge-Kutta 4 method is used to forecast trajectories for every pixel based on the forecasted flow fields and accordingly the images are reconstructed at the forecasting lead time [35]. A performance assessment used to evaluate the model using the cross-correlation value between observed and forecasted images FCC (Forecasting Cross correlation). Table 1 summarizes the Forecasting Cross correlation (FCC) values between the acquired satellite images and forecasted images at the same times and the average forecasting accuracy of the model. It should be noted that normal (i.e., natural) maximum correlation between any successive acquired images fall within (0.6-0.8) depending on how cloud patterns change between successive images. 
Table (1) FCC (Forecasting Cross correlation) assessment

\begin{tabular}{|c|c|c|c|}
\hline FCC (Absolute) & $\begin{array}{c}30 \text {-min- } \\
\text { forecasts }\end{array}$ & $\begin{array}{c}60-\text { min- } \\
\text { forecasts }\end{array}$ & $\begin{array}{c}120 \text {-min- } \\
\text { forecasts }\end{array}$ \\
\hline $\begin{array}{c}\text { Accuracy (FCC Percentage of } 0.7 \\
\text { maximum natural correlation) }\end{array}$ & 0.64 & 0.52 & 0.38 \\
\hline & $91 \%$ & $74 \%$ & $54 \%$ \\
\hline
\end{tabular}

\section{Conclusions and Discussion}

A cloud motion detector and forecaster (CMDF) model is developed in this study. The model performs both tracking and forecasting tasks. The advantage of the CMDF tracking lies in its ability to distinguish the differential motion of clouds through both the subdivision and the spatial interpolation processes. On the other side, the novelty of the forecasting module exists in providing real time updates of the forecasts based on the local changes that occur in the velocity vectors in every pixel rather than the traditional approach of projecting the motion in space. This achieves stability in the forecasts and this what makes the model very attractive for operational real-time application. For best implementation of the forecasting module, Runge-Kutta-4 numerical integration can be used to develop trajectories from the forecasted velocity vectors [35]. This can determine future trajectories of all pixels in the satellite domain. Accordingly, the image can be re-constructed in the future.

The good selection of the size of the square boxes (i.e., divisions or sub-domains) can maximizes the accuracy of the model. Of course small box size (i.e., more boxes) can better detect the spatial variability of the flow field, however, the smaller the box is, the more chance for ambiguity in matching it with itself in the next image (i.e., increased chance for mismatch and accordingly chance of wrong estimation of the optimum displacement). Hence, a compromise has to be made. The box size must be small enough to give more boxes to capture the spatial variability and yet should be large enough to have a distinguished pattern inside that can be matched with the subsequent image. For example, considering a small box in a large fully white or black area, then one will not be able to find a match for it because simply a maximum correlation can be found everywhere with every displacement (no details to match). Hence the selection of the box size should be based on the predominant storm size in the region (should capture the minimum dimension of a storm system). In addition, a constraint must be used to select only boxes that contain a distinguished (i.e., having high variability) boxes. This constraint excludes any box that has no variability inside (any box that has a coefficient of variation $\mathrm{CV}<0.2$ is excluded from the analysis). Actually, this is an excellent constraint because it excludes black boxes (i.e., clear sky areas). This cutoff value (i.e., 0.2) is selected based on a pre-analysis that was done on the cloud data. In other words, any box having CV less than 0.2 will be difficult to match because it is either very white or very black and can produce noisy displacements that may affect the results if considered in the spatial interpolation. Studying the optimum selection of box size and the fine tuning of the coefficient of variation cutoff value are also very good topics for future studies.

\section{Acknowledgment}

The European Organization for the Exploitation of Meteorological Satellites (EUMETSAT) and the Egyptian Meteorological Authority (EMA) are acknowledged for providing the satellite images used in this research. 


\section{References}

[1] Orlanski, I, “A rational subdivision of scales for atmospheric processes,", Bull. Amer. Meteor. Soc., 1975, Vol.56, pp. 527-530.

[2] Lushine, J. B., Convective growth and movement as seen 604 from GOES-1. Picture of the Month. Mon. Wea. Rev., 1976, Vol.104, pp. 1449-1450.

[3] Browning, K. A., Nowcasting, Academic Press, 1982, pp. 256.

[4] Browning, K.A., and Collier, e.G., "Nowcasting of precipitation systems". Rev. Geophys., 1989, Vol. 27(3), pp. 345-370.

[5] Hamill, Thomas M. and Thomas Nehrkorn, "A short-term cloud forecast scheme using cross correlations". Weather and Forecasting, 1993, Vol. 8(4), pp. 401- 411.

[6] Wilson, James W., N. Andrew Crook, Cynthia K. Mueller, Juanzhen Sun, and Michael Dixon, "Nowcasting thunderstorms: a status report". Bulletin of the American Meteorological Society, 1998, Vol. 79(10), pp. 2079-2099.

[7] Pierce, C.E., P.J. Hardaker, C.G. Collier, and C.M. Haggett, "GANDOLF: A system for generating automated nowcasts of convective precipitation". Meteorological Applications, 2000, Vol. 7, pp. 341-360.

[8] Wilson, J. W., Movement and predictability of radar Echoes. Report, U.S Weather Bureau Contract CWB-11093, the Travelers Weather Research Center, Hartford, Connecticut, 1966.

[9] Zawadzki, I. I., "Statistical properties of precipitation patterns". J. Applied Meteorology, 1973, Vol. 12, pp. 459-472.

[10] Austin, G. L., and Bellon, A., "The use of digital weather record for short-term precipitation forecasting". Qt. J. R. Meteorol. Soc., 1974, Vol.100, pp. 658-664.

[11] Hill, F. F., Whyte, K. W., and Browing, K. A., "The contribution of a weather radar network for forecasting frontal precipitation; a case study". Meteor. Mag., 1977, Vol. 106, pp. 68-89.

[12] Li, L., Schmid, W., Joss, J., " Nowcasting of motion and growth of precipitation with radar over a complex orography". J. Applied Meteo., 1995, Vol. 34, pp. 1286-1300.

[13] Wilk, K. E., and Gray, K. C., "Processing and analysis techniques used with the NSSL weather radar system". Preprint volume 14, Conf. on Radar Meteorology. American Meteorological Society, Boston, Massachusetts, 1970, pp. 369-374.

[14] Barclay, P.A, and Wilk, K.E. "Severe Thunderstorm Radar Echo Motion and Related Weather Events Hazardous to Aviation Operations". ESSA Technical Memorandum No. ERLTM-NSSL 46, 1970.

[15] Zittel, W. D., "Computer Applications and Techniques for Storm Tracking and Warning". Preprint volume, $17 \mathrm{~h}$ con! on radar meteorology, Seattle, Washington. American Meteorological Society, Boston, Massachusetts, 1976, pp.514-52.

[16] Bellon, D.E, and Austin, G.L., "SHARP (Short-term automated radar prediction) on a real time test". 17th Conf. on radar meteor., Seattle, Washington. American Meteorol. Soc., Boston, 1976, pp. 522-525.

[17] Bjerkaas, C.L., and Forsyth, D. E., An automated real-time storm analysis and storm tracking program (WEATRK). AFGL-TR-80-0316, Air Force Geophysics Laboratory, 1980.

[18] Panofsky, Hans A. and Glenn W. Brier, Some Applications of Statistics to Meteorology. Pennsylvania State University, first edition, 1958.

[19] Hilst, G. R. \& Russo, J. A., "An objective extrapolation technique for semi-conservative fields with an application to radar patterns", Tech. Memo 3, The Travelers Research Center, Hartford, CT, (1960), pp. 34.

[20] Noel, T. M. \& Fleisher, A. The linear predictability of weather radar signals, Research Rep. 34, Department of Meteorology, M.I.T., Available from Library, Massachusetts Institute of Technology, 77 Massachusetts Ave., Cambridge, MA 02139, (1960), pp. 46. 
[21] Russo, J. A. \& Bowne, N. E., "Linear extrapolation as a meteorological forecast tool when applied to radar and cloud ceiling patterns", Proceedings of the ninth weather radar conference, Kansas City, Mo., October 23-26, 1961.

[22] Kessler, E. \& Russo, J. A. "Statistical properties of weather radar echoes", Proceedings 10th Weather Radar Conference, Washington, D.C., American Meteorological Society, Boston, Massachusetts, (1963), pp. 25-33.

[23] Kessler, E. "Computer program for calculating average lengths of weather radar echoes and pattern bandedness", Journal of Atmospheric Science, (1966), Vol. 23, pp. 569-574

[24] Leese, J. A., C. S. Novak, and B. B. Clark, "An automated technique for obtaining cloud motion from geosynchronous satellite data using cross correlation." J. Appl. Meteor., 1971, Vol. 10, pp. 118-132

[25] Rinehart, R. E. \& Garvey, E. T. "Three-dimensional storm motion detection by conventional weather radar", Nature, (1978), Vol. 273, pp. 287-289.

[26] Browning, K. A., "Local weather forecasting", Proceedings of the Royal Society of London, Series A, (1980), Vol. 371, No. 1745, pp. 179-211.

[27] Lin, Y., Ray, P. \& Johnson, K., "Initialization of a modelled convective storm using Doppler radar derived fields", Monthly Weather Review, (1993), Vol. 121, pp. 27572775

[28] Gad, Mohamed. "Real-Time Rainfall Estimation and Prediction". Open Access Dissertations and Theses. Paper 1484, 2002.

[29] Horne, Matthew P Van., "Short-Term Precipitation Nowcasting for Composite Radar Rainfall Fields". Massachusetts institute of technology, 2003, pp.17-19.

[30] Zahraei, A., Hsu, K. lin, Sorooshian, S., Gourley, J. J., Lakshmanan, V., Hong, Y., \& Bellerby, T., Quantitative Precipitation Nowcasting: A Lagrangian Pixel-Based Approach. Atmospheric Research, (2012), Vol. 118, pp. 418-434.

[31] Otsuka, S., Tuerhong, G., Kikuchi, R., Kitano, Y., Taniguchi, Y., Ruiz, J. J., Miyoshi, T. "Precipitation Nowcasting with Three-Dimensional Space-Time Extrapolation of Dense and Frequent Phased-Array Weather Radar Observations". Weather and Forecasting, (2016). Vol.31(1), pp. 329-340.

[32] Chen, Z. Q., and Kavvas, M.L., "An automated method for representing, tracking and forecasting rain fields of severe storms by doppler weather radars". Journal of Hydrology, 1992, vol. 132, pp.179-200.

[33] Brown, RG., Smoothing, forecasting and prediction of discrete time series. Prentice Hall Inc., Englewood Cliffs, NJ, 1963.

[34] Gad, M.A., Soussa, H., \& Essa, M, "Developing Geographical Information System for the Estimation and Analysis of Rainfall Fields over Egypt Using METEOSAT Images." ITI Fifth Arab Conference on Geographical Information Systems, Cairo, Egypt. 2004, pp. 293-321.

[35] Gad M.A., El-Sharkawy, S.F.. "A Numerical Model for Real-Time Tracking and Forecasting of Cloud Movements on Weather Satellite Images". Research paper (under preparation). 


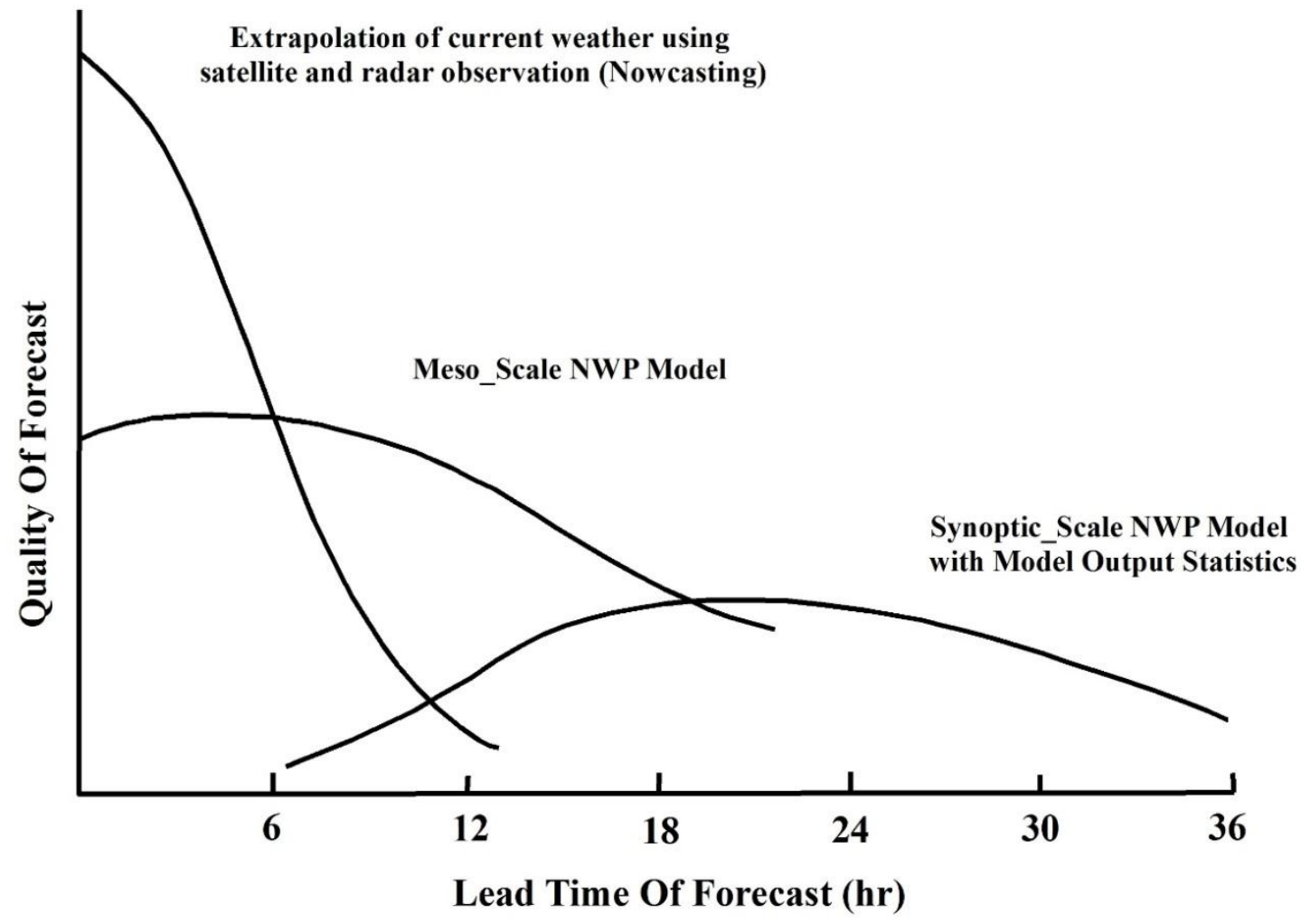

Fig. (1) A schematic diagram showing the different precipitation forecasting models. The figure was abstracted from [4]. Note that nowcasting models produce superior forecasts up to lead times 3 hours and the NWP models are better for 6-36 hours lead times.

Image at Time(t-1) Image at time (t)

Two successive satellite images

1. Define the extent of a certain box k.

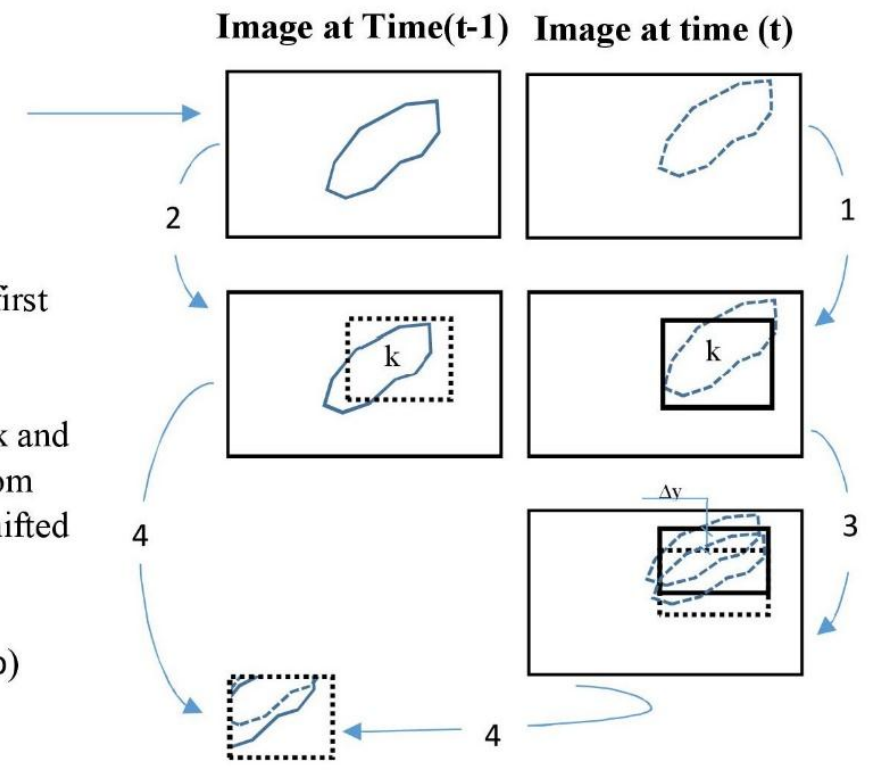

3. Shift this extent by any grid length ( $\mathrm{dx}$ and dy) and retrieve the array of values from the next image (at time $t$ ) inside the shifted extent.

4. Calculate the correlation coefficient $(\rho)$ between $\operatorname{Grid}_{(t-1)}$ and $\operatorname{Grid}_{(\mathrm{t})}$.

Fig. (2) The methodology of cross-correlation between two successive satellite images. 


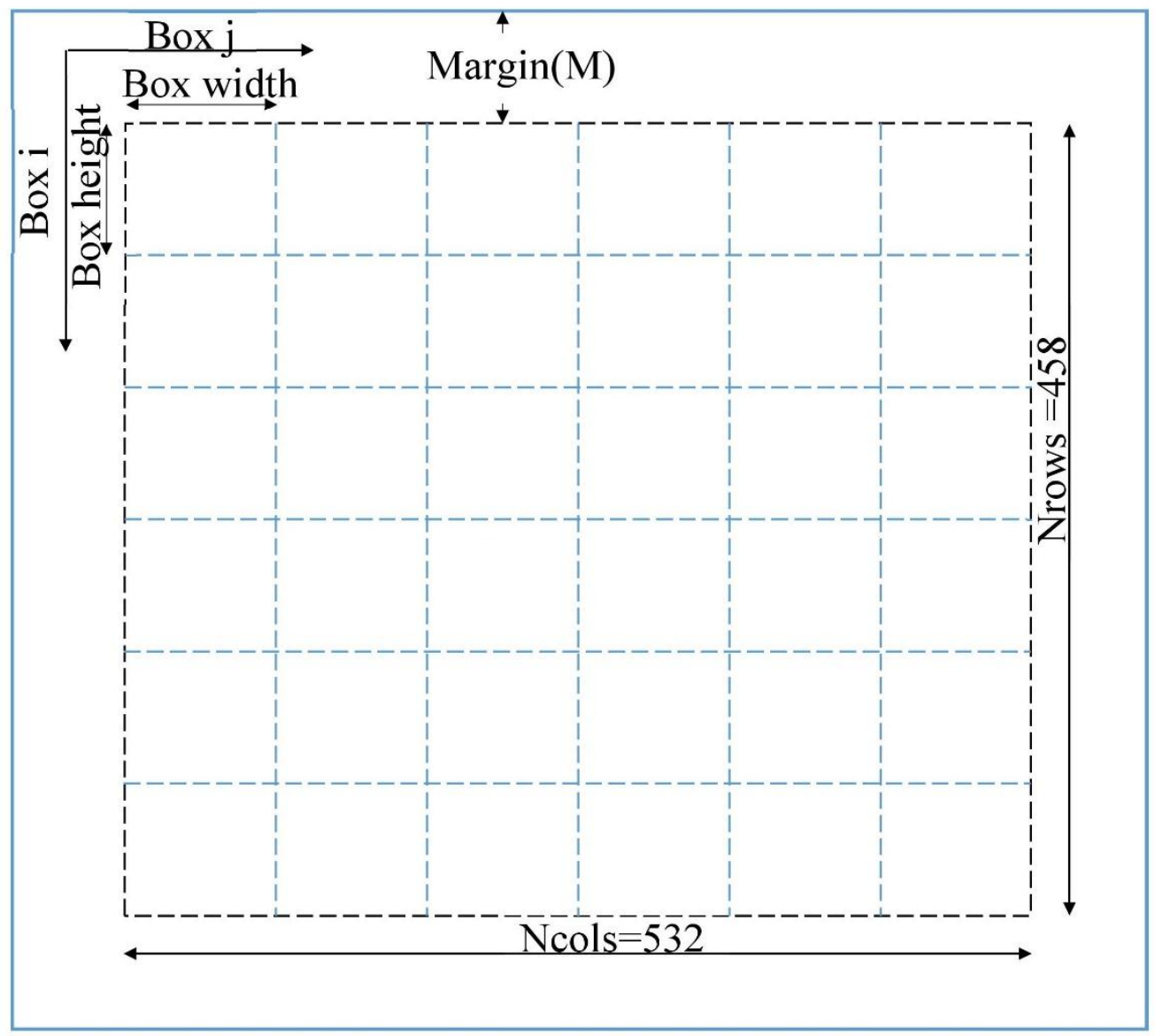

Fig. (3-a) The satellite domain (i.e., grid) is divided into number of square boxes. 


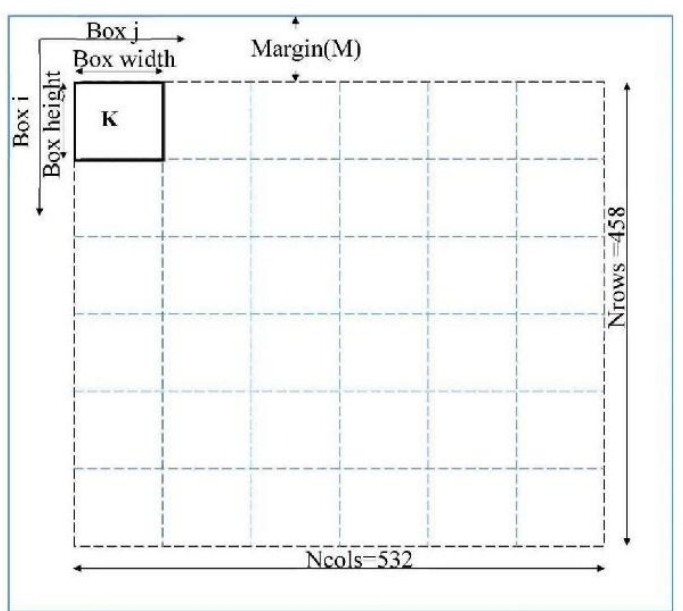

$\operatorname{Grid}_{(\mathrm{t}-1)}$ at Time(t-1)

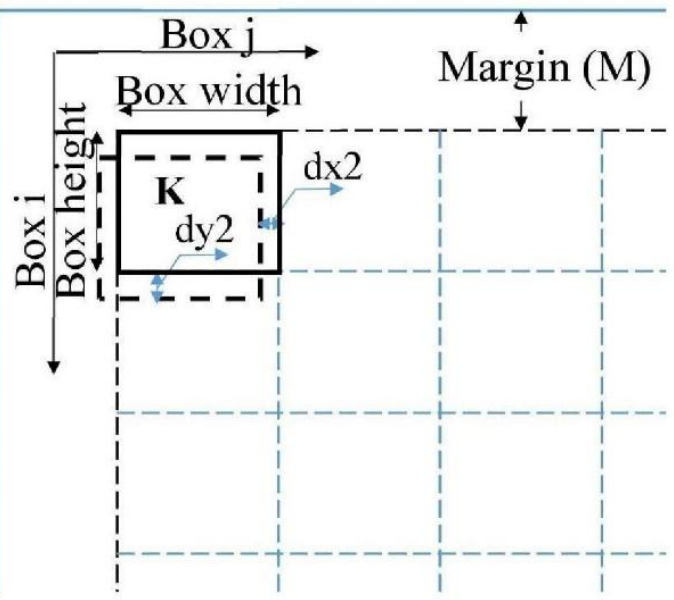

Shifted Box K of $\operatorname{Grid}_{(\mathrm{t})}$ at Time $(\mathrm{t})$ by shift $\left(\mathrm{dx}_{\mathrm{t}}, \mathrm{dy}_{\mathrm{t}}\right)$ at $\mathrm{i}=2$

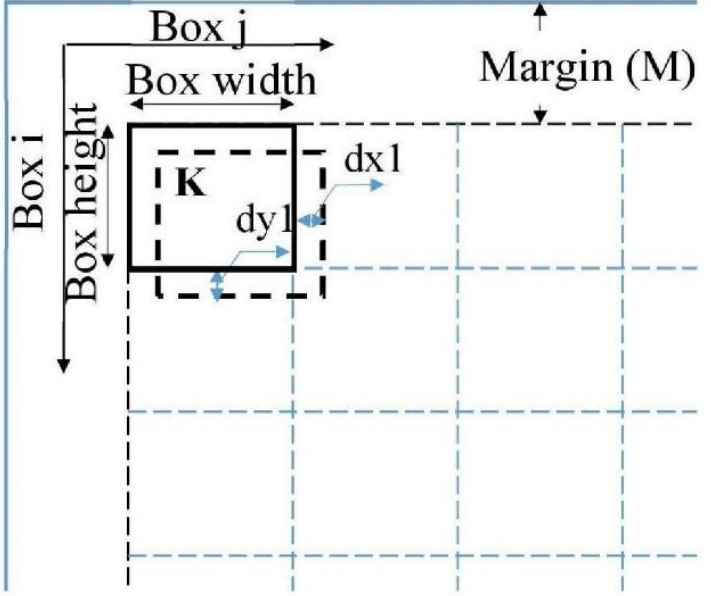

Shifted Box K of Grid $_{(t)}$ at Time (t) by shift $\left(\mathrm{dx}_{\mathrm{t}}, \mathrm{dy}_{\mathrm{t}}\right)$ at $i=1$

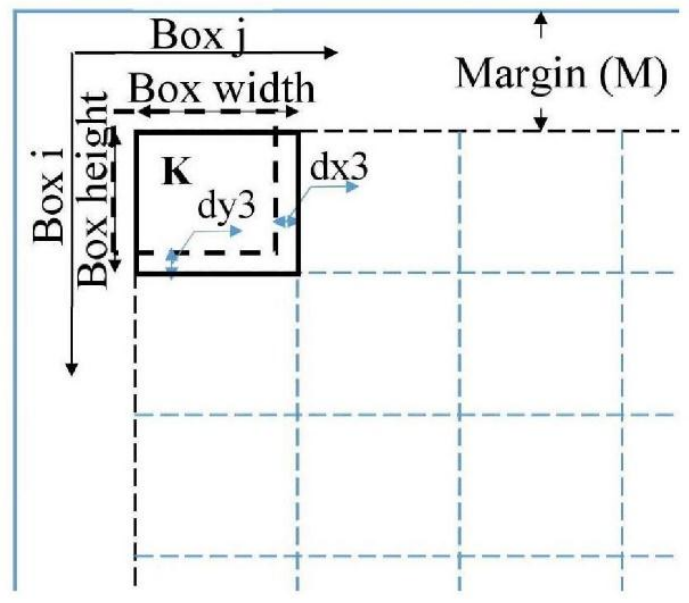

Shifted Box K of Grid $(t)$ at Time $(\mathrm{t})$ by shift $\left(\mathrm{dx}_{\mathrm{t}}, \mathrm{dy} \mathrm{t}\right)$ at $i=3$

Fig. (3-b) Sub-dividing the satellite image into boxes in the tracker to perform the cross-correlation separately for every box. The tracker finds the optimum shift for each box by attempting all possible shifts. 


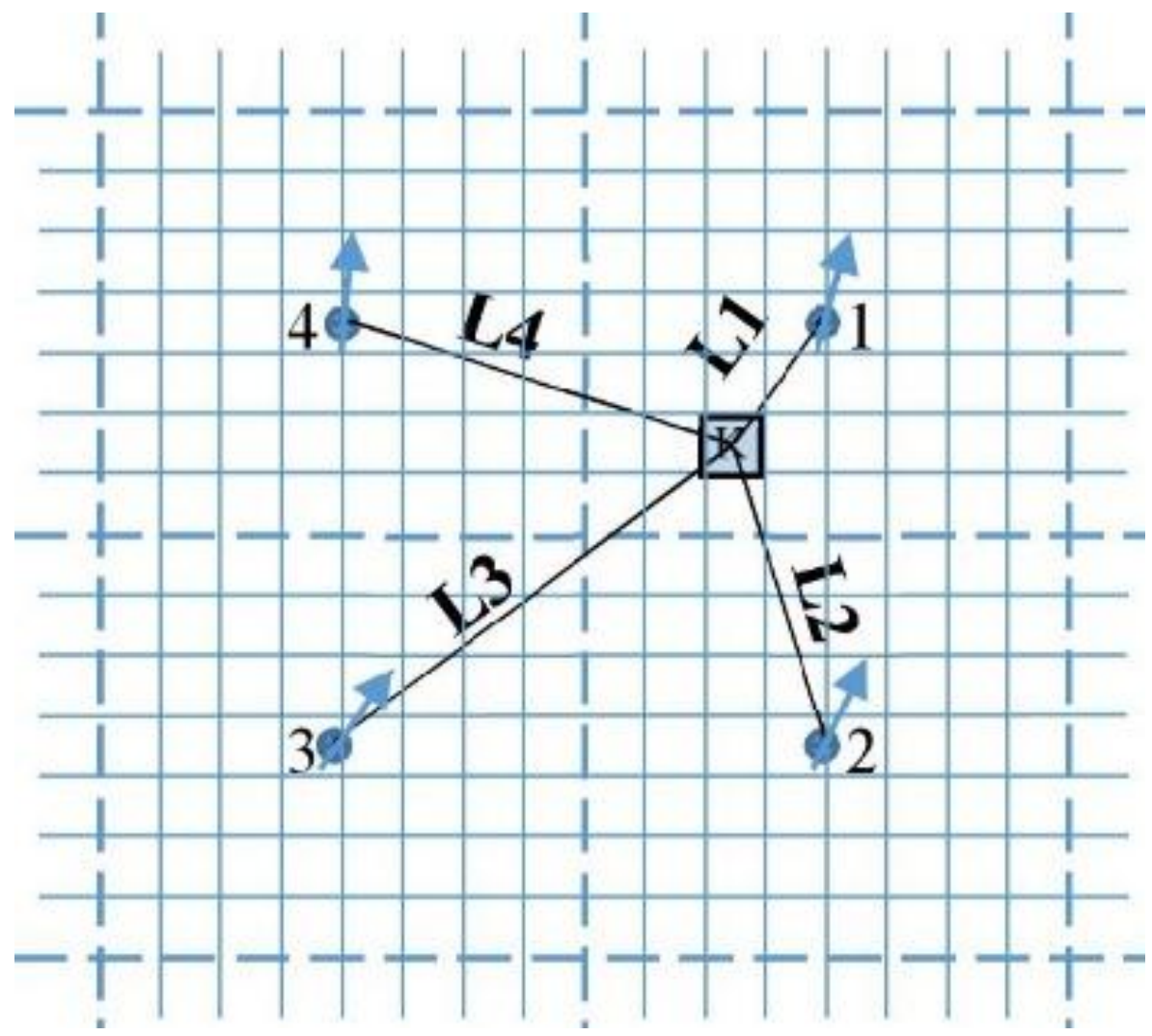

Fig. (4) Spatial interpolation of the velocity vectors from the box centers into the grid pixel. The velocity vectors are interpolated from the centers of the boxes into the high resolution pixels (i.e., grid cells) using IDW spatial interpolation method. 


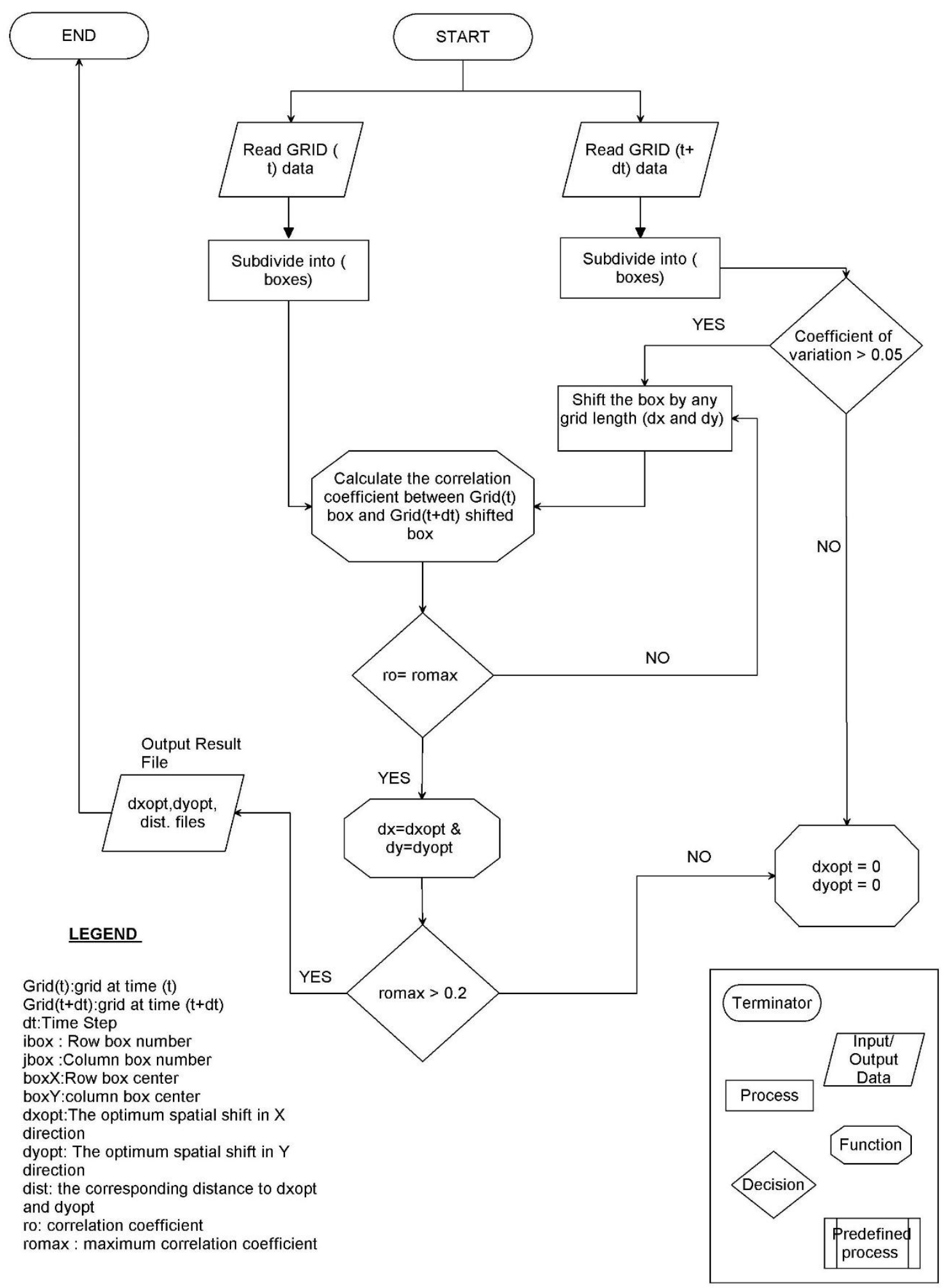

Fig. (5) Details of the CMD module. 


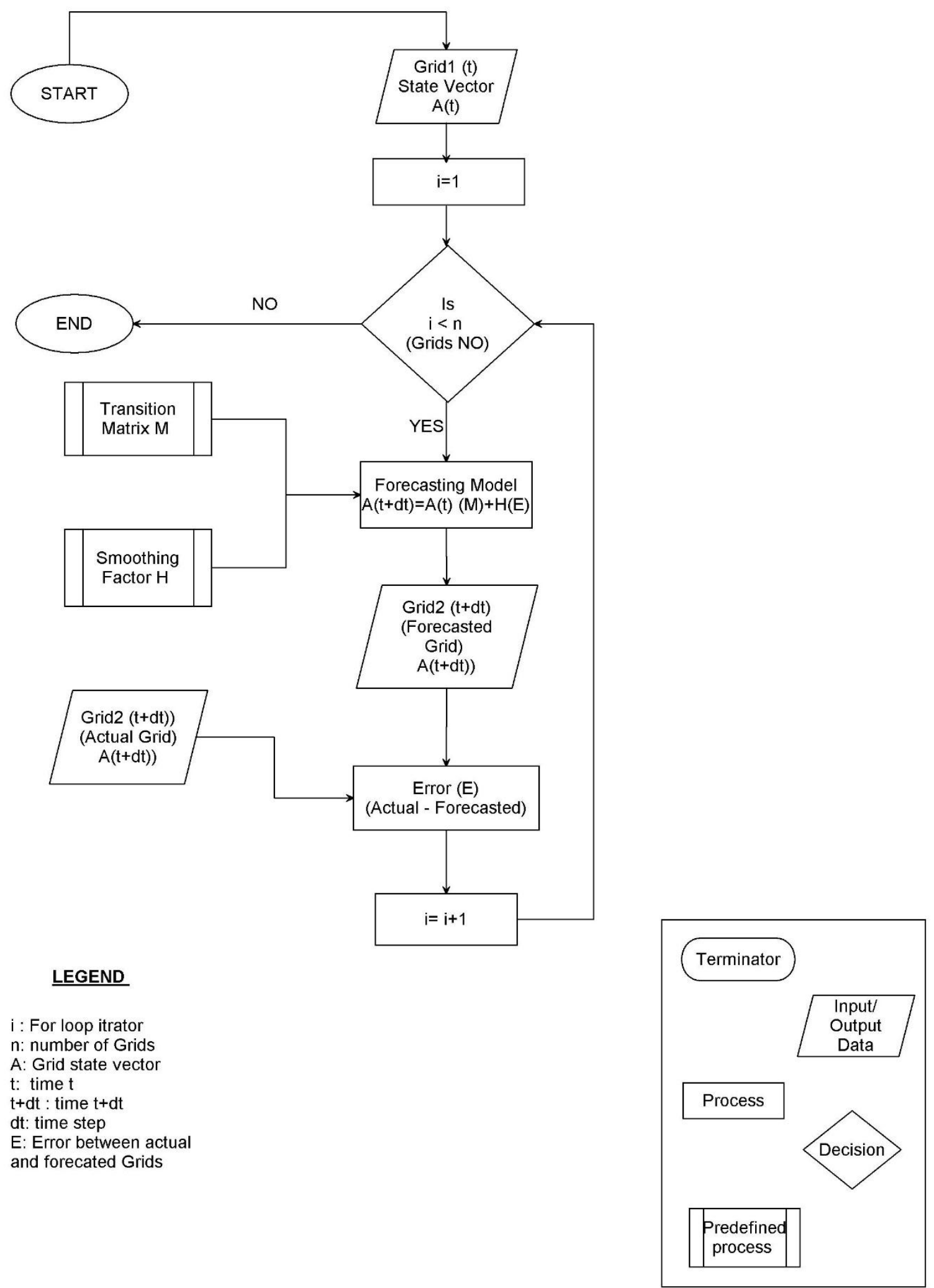

Fig. (6) Details of the CMF module. 


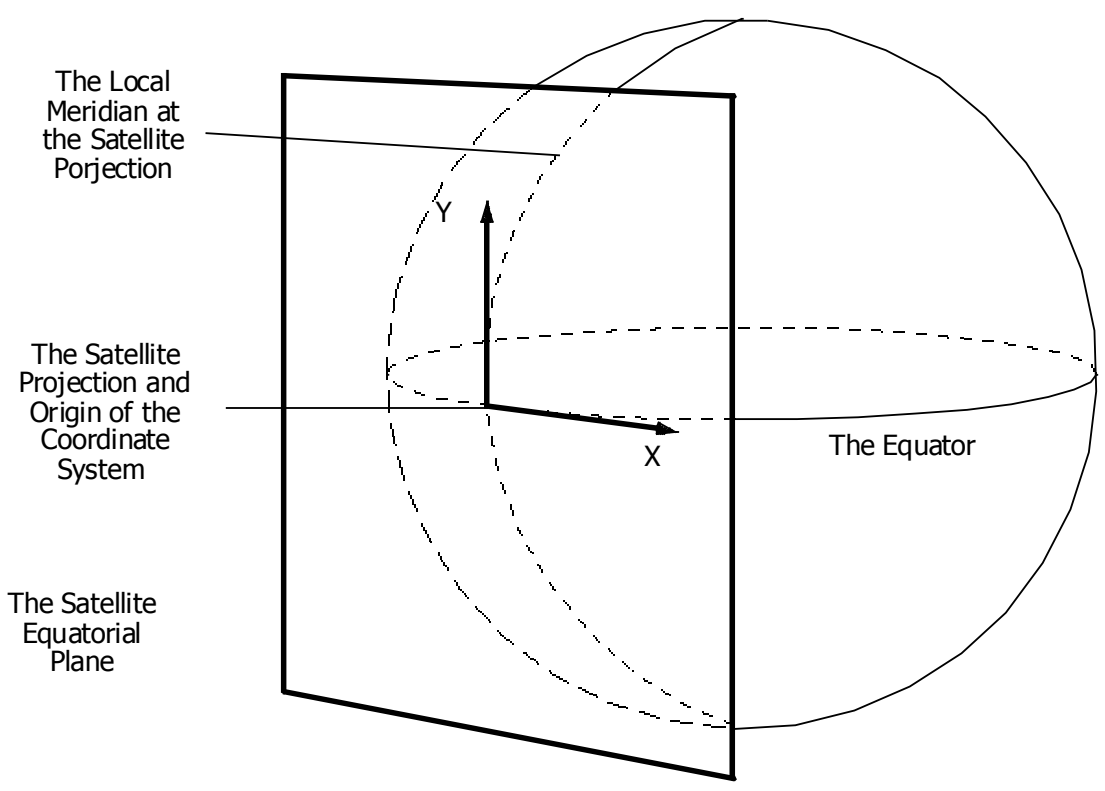

Fig. (7) The satellite near-vertical perspective coordinate system. Rays of the projection originates from the sensor at $35800-36000 \mathrm{~km}$ altitude towards any detection and intersects the satellite plan at its corresponding projection. [34] 
GOES12 image at 20110606114500 UTC geo-referenced in a vertical perspective projection (central longitude $=50 \mathrm{~W}$, satellite height $=35800 \mathrm{~km}$ )

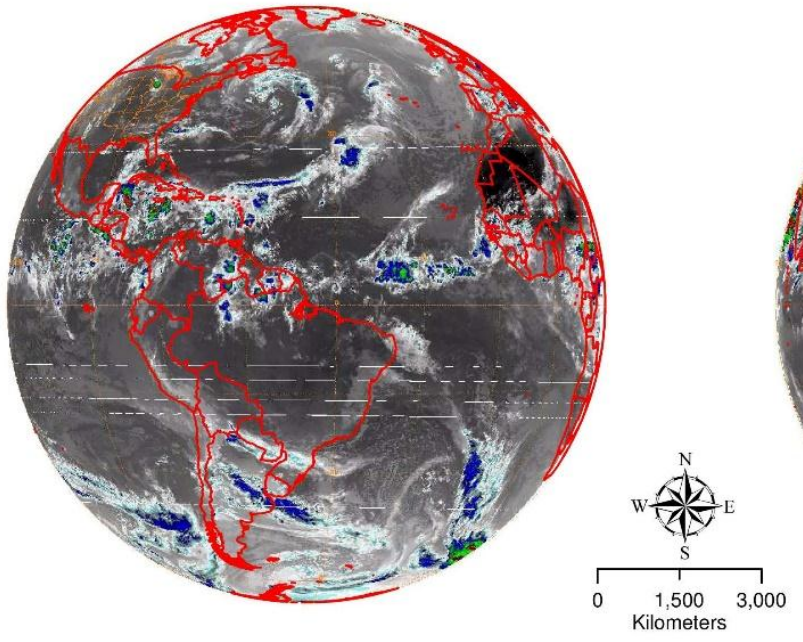

MTSAT image at 20110606114500 UTC

geo-referenced in a vertical perspective projection (central longitude $=140 \mathrm{E}$, satellite height $=35800 \mathrm{~km}$ )

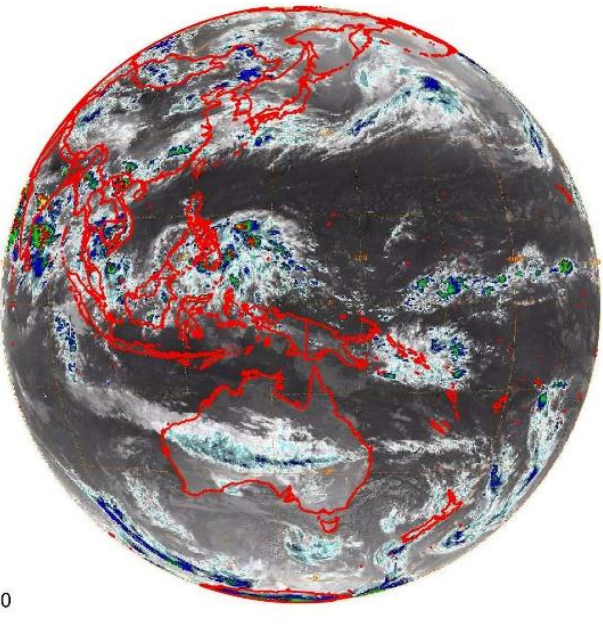

Fig. (8-a) Sample 2 IR images from GOES12 and MTSAT at 20110606114500 UTC geo-referenced each in its separate vertical satellite perspective coordinate system.

Refer to section 4.1 (Data preprocessing)

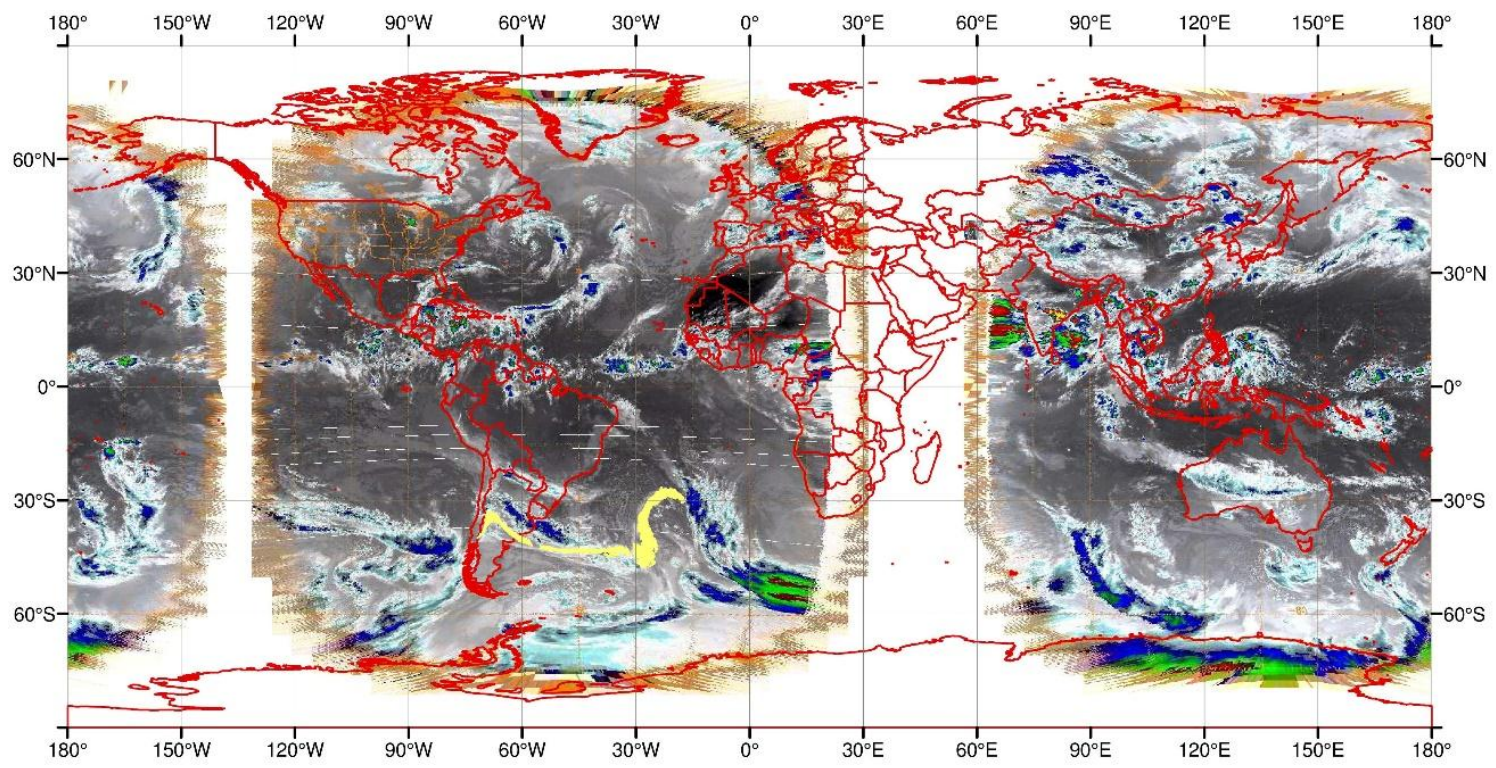

Fig. (8-b) The two sample IR images at 20110606114500 UTC after re-projection in the World Geodetic System 1984 (WGS1984) common geographic coordinate system. Refer to section 4.1 (Data preprocessing) 


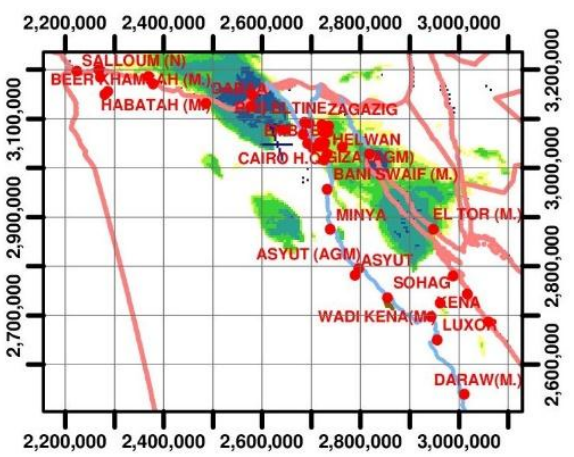

199411022000 UTC

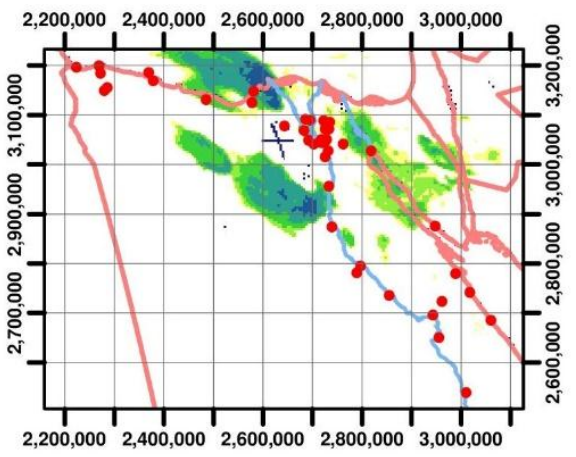

199411022130 UTC

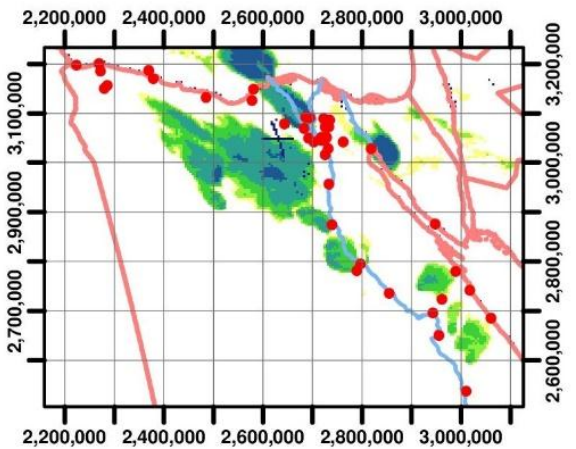

199411022330 UTC

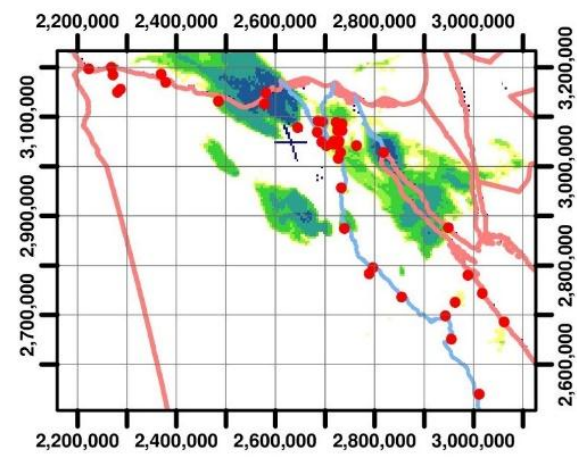

199411022030 UTC

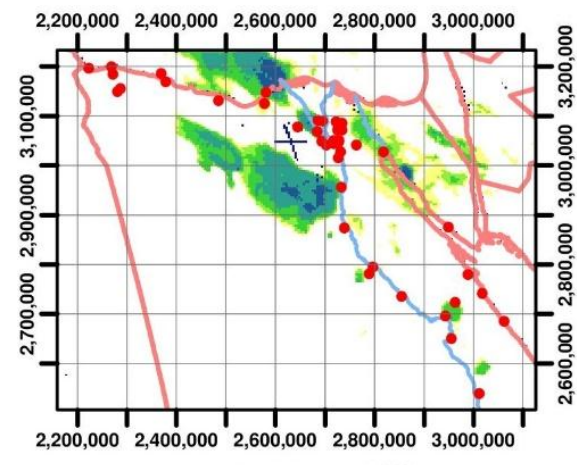

199411022230 UTC

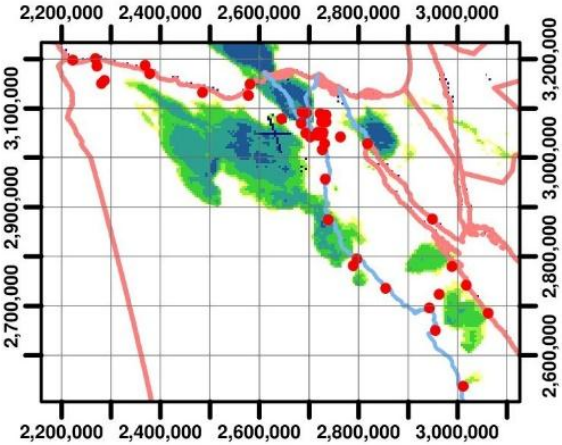

19941102400 UTC

HEAVY RAIN CHANCE

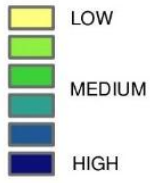

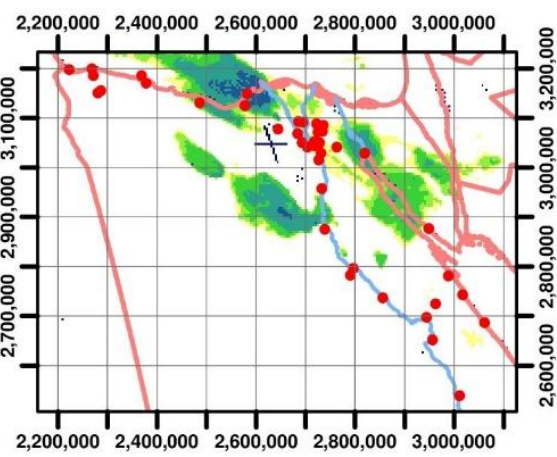

199411022100 UTC

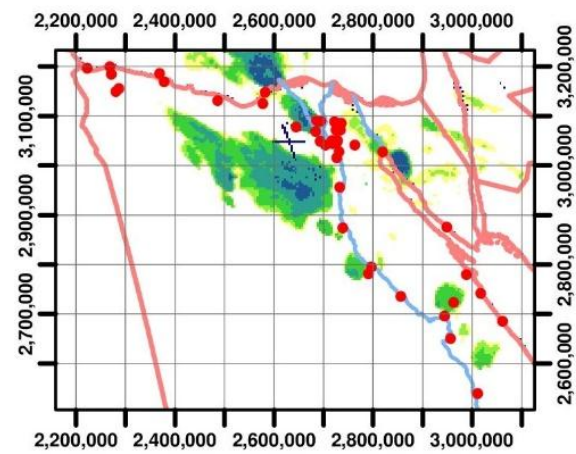

199411022300 UTC

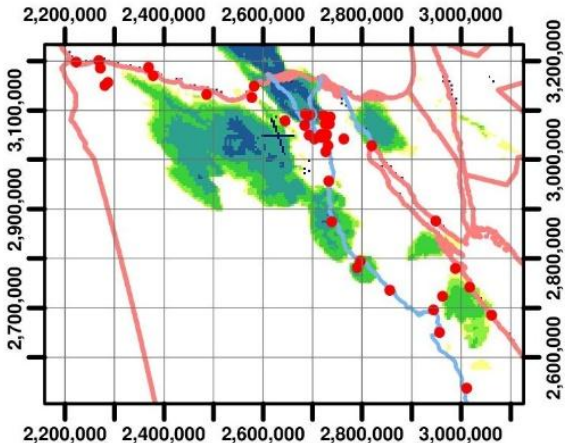

199411030000 UTC

Fig. (9) Sample Meteosat-9 pre-processed sequences of images (2/11/1994). The coordinate system is near-vertical coordinate system (Lat $=0$, Long $=0$ ) where the Satellite is at $35800 \mathrm{~km}$ above the equator. The time separation between images is 30 minutes. The images were preprocessed as described in the section (4.1). The brightness in images is classified into 3 classes:

(1) Low (for pixel numbers <195);

(2) Medium (for pixel number between 195\&215);

(3) High (for pixel numbers $>215$ ). 


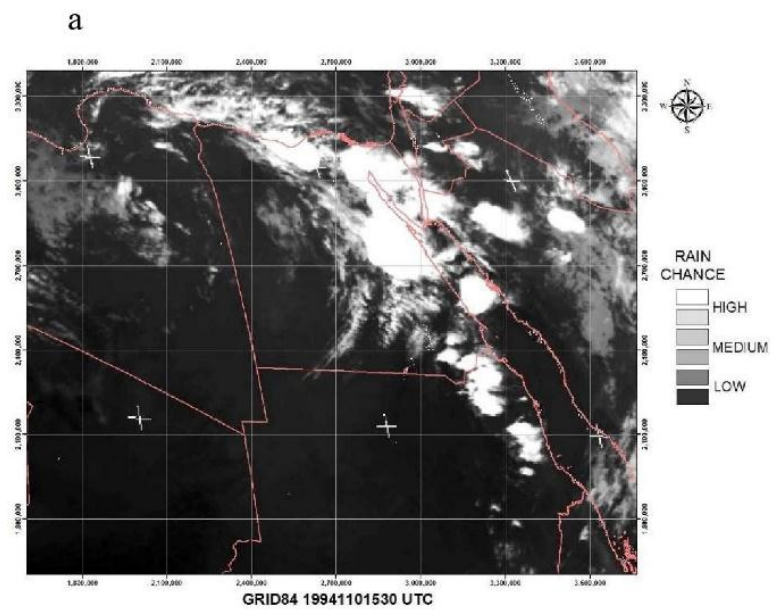

b
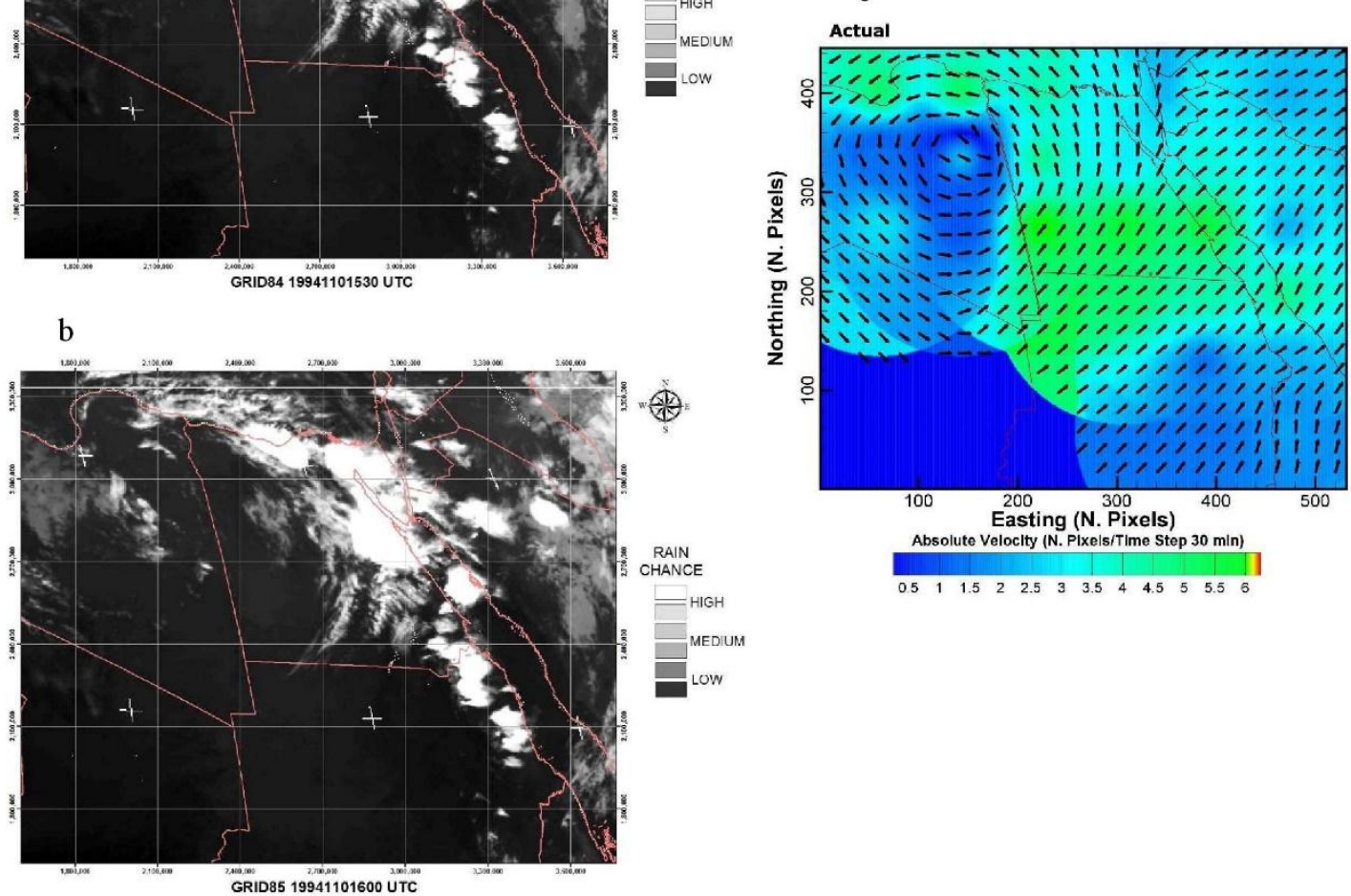

Figure (10-a) Sample two successive images $(a, b)$ and the calculated velocity field (c) resulted from CMD module. 

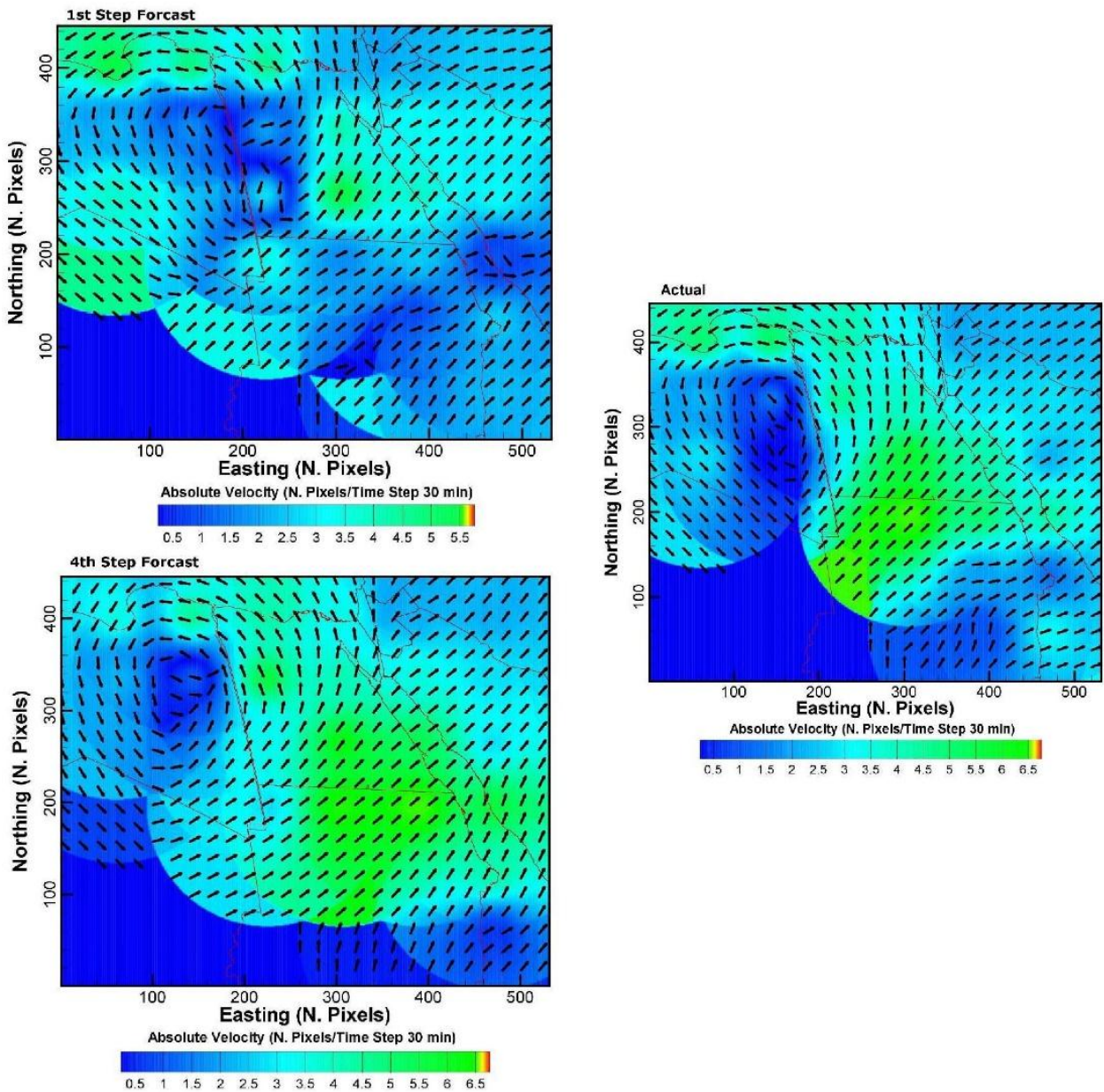

Figure (10-b) Comparison between the actual velocity field and the forecasted velocity fields. The forecasted velocity fields are those that have been forecasted in the past using 30-min (i.e., 1 step forecast) and 120 min (4 steps forecast) lead times. 